\title{
Der kulturelle Nachweis von Tuberkelbazillen im Nasenspülwasser und seine Bedeutung.
}

\author{
Von \\ Risyun Yasumoto. \\ (安 本 利 俊)
}

(Aus der Medizinischen Klinik der Kaiserlichen Tohoku-Universität zu Sendai, Leiter: Prof. Dr. T. Kumagai und Prof. Dr. T. Ebina)

\section{Einleitung.}

Der Infektionsprozess der Tuberkulose ist noch nicht ganz geklärt, obwohl sich seit $\mathrm{R}$. Koch viele Autoren damit beschäftigt haben. Im allgemeinen nimmt man aber an, dass bei dieser Krankheit die Luftinfektion eine bedeutende Rolle spielt : so die Cornet ${ }^{11}$ sche Staubinhalationstheorie, die Flüg g $\mathrm{e}^{2)}$ sche Tropfeninhalationstheoric usw. Auf Grund dieser Theorien sind verschiedene prophylaktische Methoden entwickelt worden. Nach G. Deycke ${ }^{3)}$ haften eingeatmete Tuberkelbazillen zunächst an der Nasenund Mund-schleimhaut und gelangen dann in die Halsdrüsen oder durch Verschlucken in den Verdauungstraktus. Darum gilt es im allgemeinen als Tatsache, dass Nase und Mund eine ideale Eintrittspforte für Tuberkelbazillen sind. Dass die Infektion nach der Art der Tropfeninhalationstheorie vor sich geht, stellt eine wichtige soziale Frage dar; über Methoden der Infektions-vorbeugung, den Grad der Ansteckungsgefahr und die Empfänglichkeit liegen viele Mitteilungen vor.

Der Gefahr der Tropfen-infektion sind Ärzte, Pflegerinnen, Wärterinnen usw. die mit an offener Lungentuberkulose Erkrankten in Berühtung kommen, am meisten ausgesetzt. Der Nachweis von Tuberkelbazillen in der Nasenhöhle solcher Gefährdeten wurde zuerst 1894 von einєm Franzosen namens J. Straus ${ }^{4}$ unternommen. Er sammelte rämlich mit eincm Glasstäbchen Staub, Kruste, Schleim usw. aus der Nasenhöhle Nichttuberkulöser und von Ärzten sowie Pflegerinnen, welche in Zimmern tuberkulöser Kranker tätig waren, und impfte das Material nach Waschen mit Bouillon oder sterilem Wasser ins Peritoneum von Meerschweichen. Dadurch gelang ihm die Ansteckung der Versuchstiere. LeNoir und J. 
Camus ${ }^{5)}$ machten ähnliche Versuche, indem sie Meerschweinchen Material, in dem sich Nasenschleim von Ärzte, Pflegerinnen usw. befand, unter die Haut spritzten, konnten aber keine positiven Fälle von Tuberkulose-Infektion erzielen. A. Meyer ${ }^{6)}$ verfolgte das Problem der Spontaninfektion ebenso vergeblich, indem er Tuberkelbazillen aus Gurgelwasser der Pflegerinnen und Tuberkuloselaboratoriums-Gehilfen zu züchten versuchte oder das Versuchsmaterial Meerschweinchen einimpfte.

Während die Berichte über den pathologisch-histologischen Nachweis von Tuberkelbazillen oder tuberkulösen Veränderungen zur Diagnose der Nasentuberkulose zu zahlreich sind, um einzeln angeführt werden zu können, liegen doch bei dieser Tuberkulose-form nur wenige Angaben über den Nachweis von Tuberkelbazillen durch Kultur oder Tierimpfung vor. Ja, es gibt fast keine über einen Versuch, in dem man Tuberkelbazillen aus dem Staub von Zimmern tuberkulöser Kranker kultivierte und die Art und Weise der spontanen Ansteckung mit Tuberkelbazillen untersuchte. Mit besonderer Berücksichtigung dieses Problems haben wir an Ärzten, Pflegerinnen, Schülerinnen der Schwesterschule und Wärterinnen, welche in Krankenzimmern von an offener, schwerer Tuberkulose Erkrankten tätig waren, die folgenden Versuche ausgeführt, um die Tuberkelbazillen in der Nasenund Mundhöhle nachzuweisen und die Bedeutung dieses Nachweises klarzustellen.

\section{Züchtung der Tuberkelbazillen aus Nasenspülwasser.}

1. Versuchsmaterial.

Untersucht wurden vorwiegend die Pflegerinnen, Schülerinnen der Pflegerinnenschule sowie Wärterinnen, an denen Ka to ${ }^{7)}$ seit April 1941 jährlich zweimal (Frühling und Herbst) die Röntgenuntersuchung der Lunge, Tuberkulinreaktion und die Messung der Senkungsgeschwindigkeit der roten Blutkörperchen und monatlich 1-4mal die Züchtung der Tuberkelbazillen aus Sputum usw. durchgeführt hatte. Diese Personen arbeiteten in Zimmern von an offener, schwerer Lungentuberkulose Erkrankten. Ferner wurden die Versuche an Tuberkulösen und Nichttuberkulösen unserer Klinik und an Ärtzen, die Tuberkulose behandeln, durchgeführt.

2. Versucbsmetbodin.

a) Nasenspülung.

Als Spülapparat wurde die sog. englische Pumpe benutzt, und zur Spülung wurde eine sterile $0,9 \%$ ige Kochsalzlösung angewendet, die voher auf $28-30^{\circ} \mathrm{C}$ gebracht worden war. Den Kopf der Versuchsperson hinläglich nach vorn gebeugt, brachte man die Olive an der Spitze des Gummischlauchs in den einen Naseneingang und spritzte das Spülwasser langsam ein. Mit 
einem sterilen Reagenzglas (Länge : $30 \mathrm{~cm}$, Durchmesser : $2,5 \mathrm{~cm}$, Kapazität: $100 \mathrm{ccm}$ ), welches in den anderen Naseneingang gesteckt war, wurden ungefäht 70-80 ccm Spülwasser entnommen. Dann wurde die Olive in den Naseneingang, in dem sich vorher das Reagenzglas befunden hatte, und ein Reagenzglas in denjenigen, in den vorher die Olive eingeführt worden war, eingeführt. Bei diesem Verfahren wurden wieder 70-80 ccm Spülwasser entnommen. Dabei liess man die Versuchsperson durch den Mundatmen und verbot das Sprechen. Beim Einspritzen des Spülwassers musste die Versuchsperson den Ton , $\mathrm{i}-\mathrm{i}-\mathrm{i}$ “" sprechen, um der Otitis media vorzubeugen.

Die Spülung wurde vormittags an Patienten unserer Klinik und nachmirtags an Ärzten, Pflegerinnen, Schülerinnen der Pflegerinnenschule und Wärterinnen ausgeführt.

b) Züchtung.

Ungefähr $70 \mathrm{ccm}$ des auf obengeschilderte Weise gewonnenen Nasenspülwassers wurden nach Zusatz von $10 \mathrm{ccm} 2 \%$ iger Natronlaugelösung tüchtig ungerührt. Dem so gelösten Schleim wurden dann ungefähr 3-5 $\operatorname{ccm} 2,5 \%$ igen Zinksulfats hinzugefügt; dann wurde wieder umgerührt, bis ein weisser flockiger Niederschlag von Zinkhydroxyd entstand. Nach 5-10 Minuten langem Stehenlassen wurde der Niederschlag in ein Spitzglas gegossen und mittels einer Zentrifuge von 3000 Umdrehungen pro Minute 5 Minuten lang zentrifugiert. Die überstehende Flüssigkeit wurde weggegossen und der Niederschlag wurde nach Zugabe von ungefähr $15 \mathrm{ccm} 4 \%$ iger Schwefelsäurelösung tüchtig umgerührt, ungefähr 20-30 Minuten lang bei Zimmertemperatur stehen gelassen und dann wieder 5 Minuten lang mit 3000 Umdrehungen pro Minute zentrifugiert. Nach dem Weggiessen der überstehenden Flüssigkeit wurde der Niederschlag auf 3-5 Ok a-Kata kura ${ }^{83}$ sche Phosphat-Ajinomoto-Hühnerei-Nährböden gebracht und zwei Monate lang im Brutschrank bei $37^{\circ} \mathrm{C}$ aufbewahrt. Während dieser Zeit wurde 5 mal d. h. am 10., 20., 30., 40. und 60. Kultur-Tag die Entwicklung der Kolonien untersucht. Die Differenzierung der Tubetkelbazillen und der apathogenen säurefesten Bazillen wurde sehr sorgfältig durchgeführt. Bei der Beurteilung haben wir die Färbbarkeit, die mikroskopische Form und die Eigenschaft der Bazillen sowie die Entwicklung der Kolonie zu der Zeit, in der sie sich auf dem Oka-Katakuraschen Nährboden befanden und später auf den Agar-Nährboden kamen, berücksichtigt. Wenn die Beurteilung schwer war, wurde mit Versuchen an Meerschweinchen die Virulenz der herausgezüchteten Bazillen geprüft.

3. Versuchsergebnisse.

1. Bei Personen, die der Gefahr der 'Tuberkulosenansteckung ausgesetzt sind. 
An 9 Ärzten, 25 Pflegerinnen, 38 Schülerinnen der Pflegerinnenschule und 75 Wärterinnen, insgesamt bei 147 Fällen und zwar in jedem Falle 1-4 mal haben wir die Züchtung von Tuberkelbazillen aus Nasenspülwasser durchgeführt und die Resultate gewonnen, die auf Tabelle 1 angegeben sind. Unter diesen 147 Fällen fiel nämlich die Züchtung der Tuberkelbazillen aus Nasenspülwasser nur bei 2 Personen $(1,3 \%)$, und zwar in $2(0,7 \%)$ von 293 Versuchen positiv aus, d. h. bei 1 Fall $(2,6 \%)$ von 38 Schülerinnen der Pflegerinnenschule und 1 Fall $(1,3 \%)$ von 75 Wärterinnen.

\section{Tabelle 1.}

Kulturergebnisse aus Nasenspülwasser bei den Personen, die der Gefahr der Tuberkulösenansteckung ausgesetzt sind.

\begin{tabular}{l|c|c|c|c|c|c}
\hline $\begin{array}{r}\text { Züchtung aus } \\
\text { Nasenspül- } \\
\text { wasser }\end{array}$ & Fälle & $\begin{array}{c}\text { Tuberkel- } \\
\text { bazillen }\end{array}$ & $\begin{array}{c}\text { Apathogene } \\
\text { säurefeste } \\
\text { Bazillen }\end{array}$ & $\begin{array}{c}\text { Zahl der } \\
\text { Versuche }\end{array}$ & $\begin{array}{c}\text { Tuberkel- } \\
\text { bazillen }\end{array}$ & $\begin{array}{c}\text { Apathogene } \\
\text { säurefeste } \\
\text { Bazillen }\end{array}$ \\
\hline Arzte & 9 & 0 & $2(22,2 \%)$ & 9 & 0 & $2(22,2 \%)$ \\
\hline Pflegerinnen & 25 & 0 & $14(56,0 \%)$ & 79 & 0 & $19(24,0 \%)$ \\
\hline $\begin{array}{c}\text { Schulerinnen der } \\
\text { PAlegerinnschule }\end{array}$ & 38 & $1(2,6 \%)$ & $13(34,2 \%)$ & 38 & $1(2,6 \%)$ & $13(34,2 \%)$ \\
\hline Wärterinnen & 75 & $1(1,3 \%)$ & $31(41,3 \%)$ & 167 & $1(0,6 \%)$ & $36(21,5 \%)$ \\
\hline Gesamtzahl & 147 & $2(1,3 \%)$ & $60(40,8 \%)$ & 293 & $2(0,7 \%)$ & $70(23,8 \%)$
\end{tabular}

Bei diesen zwei Fällen war die Tuberkulinrcaktion positiv, das Röntgenbild der Lunge aber normal, und die mehr als zehnmal ausgeführte Zuchtung aus Sputum fiel negativ aus. Die Kolonien der herausgezüchteten Tuberkelbazillen waren an Zahl sehr gering, d. h. bei der Shülerin der Pflegerinnenschule trat auf 5 Nährböden nur eine Kolonie und bei der Wärterin auf 3 Nährböden ebenfalls nur eine Kolonie auf.

Ferner wurden bei den Versuchspersonen aus dem Nasenspülwasser viele apathogene säurefeste Bazillen nachgewiesen. Wie man aus Tabelle 1 ersieht, wurden sie insgesamt bei $60(40,8 \%)$ von 147 Fällen und zwar in $70(23,8 \%)$ von 293 Versuchen nachgewiesen.

Hier sei kurz berichtet über das damalige Milieu, die klinischen Symptome, die biologischen Reaktionen, das Röntgenbild und den Verlauf dieser 2 Fälle mit positivem Ergebnis :

Nr. 1, K. T., 17 jährige Schülerin der Pflegerinnenschule.

Im April 1941 ist sie als Schülerin der Pflegerinnenschule in die Klinik eingetreten. Damals betrug die Tuberkulinteaktion $21 \times 20 \mathrm{~mm}$, die Senkungsgeschwindigkeit $5 \mathrm{~mm}$ pro Stunde. Die Tuberkulinreaktion betrug am 20. Mai desselben jahres $41 \times 47 \mathrm{~mm}$ und im Juli desselben Jahres $50 \times 47 \mathrm{~mm}$. Vom 1. bis zum 31 . 
Oktober desselben Jahres arbeitete sie in Zimmern tuberkulöser Kranker. Bei der Züchtung vom 16. Oktober desselben Jahres wurden Tuberkelbazillen im Nasenschleim nachgewiesen. Subjektive Beschwerden und physikalische Befunde der Lunge wurden nicht beobachtet, das Röntgenbild der Lunge wies keine Veränderungen auf, die Züchtung aus Sputum wurde von Juli 1941 bis April 1942 monatlich einmal ausgeführt, fiel aber immer negativ aus.

Das Zimmer, in dem diese Schülerin der Pflegerinnenschule arbeitete, war ziemlich klein, d. h. 6,90 m lang, 2,70 m breit und 3,23 $\mathrm{m}$ hoch, und war mit 5 an offener Tuberkulose Erkrankten belegt; bei 3 von diesen fiel die Züchtung aus Sputum positiv aus, bei einer Kranken deren Tuberkulose mit Asthma bronchiale kompliziert war, war der Ausstrich aus Sputum von Gaffky I-III. Sie warf Sputum in grosser Menge aus und hustete verhältnismässig viel. Der fünfte Fall wat ein solcher schwerer Lungentuberkulose mit mehteren Kavernen; die Kranke warf Sputum in grosser Menge aus und zeigte positive Ausfälle von Tuberkelbazillen von Gaffky VII-X. Bei ihr handelte es sich um vorgeschrittene verschlechterte Lungentuberkulose mit fortwährendem Husten.

Nr. 2, Y. H., 42jährige Wärterin.

Seit 28. September 1941 diente sie als Wärterin in Zimmern tuberkulöser Kranker. Da sie nachts $z$ wischen den Betten der Patienten schlief, am Tage allerlei persönliche Geschäfte der Kranken erledigte und das Zimmer reinigte, kam sie immer mit den Kranken in Berührung.

Schon vor der Untersuchung war die Tuberkulinreaktion positiv. März 1942 betrug sie $45 \times 45 \mathrm{~mm}$ und die Senkungsgeschwindigkeit der roten Blutkörperchen 9 $\mathrm{mm}$ pro Stunde. Am 13. April desselben Jahres fiel die Kultur der Tuberkelbazillen aus Nasenspülwasser positiv aus. Die Züchtung aus Sputum, die von April 1941 bis April 1942 monatlich viermal ausgeführt wurde, war stets negativ und die Züchtung aus Gurgelwasser, die seit April 19424 Monate lang monatlich einmal ausgeführt wurde, ebenfalls immer negativ. Ohne subjektive Beschwerde, keine physikalischen Befunde der Lunge und ohne pathologische Veränderungen im Röntgenbild; arbeitet zurzeit tüchtig.

Das Zimmer, in dem diese Wärterin arbeitete, war mit 10 Tuberkulösen belegt, von denen die Züchtung aus Sputum bei zweien negativ und bei dreien positiv ausfiel. Die 5 überigen waren von Gaffky IV-X. Einer von ihnen war ein schwer Lungentuberkulöser in Komplikation mit Asthma bronchiale, dessen Vitalkapazität klein war $(-75 \%)$ und der bald infolge grosser Hämoptoe starb.

2. Bei Nichttuberkulösen.

An 48 Nichttuberkulösen, und zwar in jedem Falle 1-4 mal, haben wir die Züchtung von Tuberkelbazillen aus Nasenspülwasser durchgeführt und in keinem der 78 Versuche fiel die Züchtung von Tuberkelbazillen aus Nasenspülwasserpositiv aus, wie aus Tabelle 2 ersichtlich ist. Die apathogenen säurefesten Bazillen aber wurden bei $7(14,5 \%)$ von 48 Fällen, und zwar in $11(14,1 \%$ ) von 78 Versuchen herausgezüchtet. (Tabelle 2) 
Tabelle 2.

Beziehung zwischen den säurefesten Bazillen im Nasenspülwasser und den Tuberkelbazillen im Sputum von Tuberkulösen und Nichttuberkulösen.

\begin{tabular}{|c|c|c|c|c|c|c|}
\hline \multirow{10}{*}{ 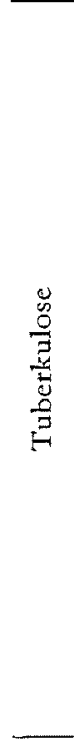 } & $\begin{array}{r}\begin{array}{r}\text { Züchtung aus } \\
\text { Nasenspül- } \\
\text { wasser }\end{array} \\
\text { Sputum }\end{array}$ & $\begin{array}{l}\text { Zahl der } \\
\text { Versuche }\end{array}$ & \multicolumn{2}{|c|}{ Tuberkelbazillen } & \multicolumn{2}{|c|}{$\begin{array}{c}\text { Apathogene säurefeste } \\
\text { Bazillen }\end{array}$} \\
\hline & fehlt & 33 & 0 & & $5(1$ & $5,1 \%)$ \\
\hline & Kultur $(-)$ & 123 & $4(3$ & $3 \%)$ & $15(1$ & $2,2 \%)$ \\
\hline & Kultur $(t)^{*}$ & 120 & $7(5$ & $8 \%)$ & $18(1$ & $5,0 \%)$ \\
\hline & Ausstrich $(-) \dagger$ & 27 & $2(7$ & $, 4 \%)$ & $1(3$, & $7 \%)$ \\
\hline & \multirow{3}{*}{ Gaffky } & \multirow{3}{*}{170} & $7(9,4 \%)$ & \multirow{3}{*}{$27(15,8 \%)$} & $15(20,0 \%)$ & \multirow{3}{*}{$25(14,7 \%)$} \\
\hline & & & $10(16,6 \%)$ & & $7(11,6 \%)$ & \\
\hline & & & $10(27,7 \%)$ & & $3(8,3 \%)$ & \\
\hline & $\begin{array}{l}\text { Gesamtzahl der } \\
\text { Versuche }\end{array}$ & $473\left\{\begin{array}{l}\hat{2} 266 \\
\$+207\end{array}\right\}$ & $40(8,4 \%)$ & $\left\{\begin{array}{l}\text { o } 13(4,9 \%) \\
\text { ㅇ } 27(13,0 \%)\end{array}\right.$ & $64(13,5 \%)$ & $\begin{array}{l}\text { of } 31(11,6 \%) \\
\text { \& } 33(16,0 \%)\end{array}$ \\
\hline & $\begin{array}{l}\text { Gesamtzahl der } \\
\text { Fälle }\end{array}$ & $142\left\{\begin{array}{ll}\hat{0} & 80 \\
0 & 62\end{array} \mid\right.$ & $28(19,7 \%)$ & $\left(\begin{array}{l}\hat{0} 12(15,0 \%) \\
\text { 우 } 16(26,8 \%)\end{array}\right.$ & $52(36,6 \%)$ & $\begin{array}{l}\text { कि } 26(32,5 \%) \\
\text { ㅇ } 26(41,9 \%)\end{array}$ \\
\hline \multirow{2}{*}{$\begin{array}{l}\text { Nicht- } \\
\text { tuber- } \\
\text { kulose }\end{array}$} & \multirow{2}{*}{ Kultur $(-)\left\{\begin{array}{l}\text { Zahl der } \\
\text { Versuche } \\
\begin{array}{l}\text { Zahl der } \\
\text { Fälle }\end{array}\end{array}\right.$} & 78 & & 0 & \multicolumn{2}{|c|}{$11(14,1 \%)$} \\
\hline & & 48 & & 0 & $7(1$ & $4,5 \%)$ \\
\hline
\end{tabular}

3. Bei Tuberkulösen.

a) Beziehung zwischen den säurefesten Bazillen im Nasenspülwasser und den Tuberkelbazillen im Sputum.

Von 142 Tuberkulösen fiel die Züchtung von Tuberkelbazillen aus Nasenspülwasser bei 28 Fällen $(19,7 \%)$, und zwar in $40(8,4 \%)$ von 473 Versuchen positiv aus. Die Beziehung zwischen diesen Resultaten und den TuTuberkelbazillen im Sputum ist in Tabelle 2 dargestellt.

Berücksichtigt man das Geschlecht, so zeigten $12(15,0 \%)$ von 80 männlichen Versuchspersonen, und zwar $13(4,9 \%)$ von 266 Versuchen, und 16 $(26,8 \%)$ von 62 weiblichen Versuchspersonen, und zwar $27(13,0 \%)$ von 207 Versuchen, positives Ergebnis.

Die Züchtung apathogener säurefester Bazillen aus Nasenspülwasser fiel bei $52(36,6 \%)$ von 142 Fällen, und zwar in $64(13,5 \%)$ von 473 Versuchen positiv aus. (Tabelle 2)

Berücksichtigt man das Geschlecht, so fiel die Züchtung apathogener säurefester Bazillen aus Nasenspülwasser bei 26 (32,5\%) von 80 männlichen 
Vetsuchspersonen, und zwar in $31(11,6 \%)$ von 266 Versuchen, und bei 26 $(41,9 \%)$ von 62 weiblichen Versuchspersonen, und zwar in $33(16,0 \%)$ von 207 Versuchen positiv aus.

Aus dem Gesagten geht hervor, dass Tuberkelbazillen im Nasenspülwasser von Tuberkulösen in einem sehr höhen Prozentstaz nachgewiesen werden und dass die Bazillenmenge sowie der Prozentsatz der positiven Ausfälle mit der Menge der Tuberkelbazillen im Sputum parallel laufen.

Das Vorhandensein und die Menge apathogener säurefester Bazillen im Nasenspülwasser ist nicht abhängig von dem Vorhandensein und der Menge der Tuberkelbazillen im Sputum.

b) Beziehung zwischen den säurefesten Bazillen im Nasenspülwasser und der Menge des Sputums (Die Sputummenge wird mit der Zahl der Auswurfe pro Tag bezeichnet)

Die Beziehung zwischen diesen Resultaten und der Sputummenge ist in Tabelle 3 dargestellt, der Prozentstaz des positiven Nachweises der Tuberkelbazillen ist bei den Tuberkulösen hoch, bei denen das Sputum in grosser Menge ausgeworfen wird, und zwar steht er im geraden Verhältnis zur Menge des Sputums, während der Prozentsatz der apathogenen säurefesten Bazillen mit der Menge des Sputums in keiner Beziehung steht.

Tabelle 3.

Beziehung zwischen den säurefesten Bazillen im Nasenspülwasser und der Menge des Sputums von Tuberkulösen.

\begin{tabular}{|c|c|c|c|}
\hline *üphtung aus & $\begin{array}{l}\text { Zahl der } \\
\text { Versuche }\end{array}$ & Tuberkelbazillen & $\begin{array}{c}\text { Apathogene säurefeste } \\
\text { Bazillen }\end{array}$ \\
\hline fehlt & 33 & 0 & $5(15,1 \%)$ \\
\hline Auswürfe (1-5) & 176 & $4(2,2 \%)$ & $17(9,6 \%)$ \\
\hline Auswürfe (6-10) & 131 & $12(9,1 \%)$ & $20(15,2 \%)$ \\
\hline Auswütfe $(11-20)$ & 68 & $11(16,1 \%)$ & $5(7,3 \%)$ \\
\hline Auswürfe $(21-30)$ & 35 & $6(17,1 \%)$ & $9(25,7 \%)$ \\
\hline Auswürfe (über 30) & 30 & $7(23,3 \%)$ & $8(26,6 \%)$ \\
\hline $\begin{array}{l}\text { Gesamtzahl der } \\
\text { Versuche }\end{array}$ & $473 \begin{cases}6 & 266 \\
7 & 207\end{cases}$ & $40(8,4 \%) \begin{cases}\hat{\delta} & 13(4,9 \%) \\
7 & 27(13,0 \%)\end{cases}$ & $64(13,5 \%)\left\{\begin{array}{l}\hat{8} 31(11,6 \%) \\
8 \quad 33(16,0 \%)\end{array}\right.$ \\
\hline $\begin{array}{l}\text { Gesamtzahl dex } \\
\text { Fälle }\end{array}$ & $142 \begin{cases}\hat{\delta} & 80 \\
q & 62\end{cases}$ & $28(19,7 \%)\left\{\begin{array}{ll}\hat{0} & 12(15,0 \%) \\
0 & 16(26,8 \%)\end{array}\right)$ & $52(36,6 \%) \begin{cases}\hat{0} & 26(32,5 \%) \\
9 & 26(41,9 \%)\end{cases}$ \\
\hline
\end{tabular}

4. Beziehung zwischen den Fällen mit positivem Ergebnis der Tuberkelbazillen-Züchtung aus Nasenspülwasser und der Nasentuberkulose. 
Bei $2(1,3 \%)$ von 147 Fällen, die der Gefahr der Tuberkulosenansteckung ausgesetzt waren, und bei $28(19,7 \%)$ von 142 Tuberkulösen wurden positive Ergebnisse der Tuberkelbazillen-Züchtung aus Nasenspülwasser erzielt. Nach den Oto-rhino-laryngologischen Untersuchungen von Hern Dr. Dazai in der Klinik für Oto-rhino-laryngologie der Kaiserlichen TohokuUniversität, zeigte keiner von den 30 Fällen mit positiven Ergebnissen tuberkulöse Veränderungen in der Nasenhöhle. Daraus kann man schliessen, dass die Nasentuberkulose sehr selten ist und dass die im Nasenspülwasser nachgewiesenen Tuberkelbazillen bei denjenigen, die der Gefahr der Tuberkulosenansteckung ausgesetzt waren, exogener Herkunft sind, während sie bei den Tuberkulösen vom eignen Sputum herrühren.

\section{Züchtung der Tuberkelbazillen aus Gurgelwasser.}

1. Versubsmaterial.

Die Züchtung der Tuberkelbazillen aus Gurgelwasser wurde gleichzeitig mit der aus Nasenspülwasser durchgeführt. Untersucht wurden Pflegerinnen, Schülerinnen der Pflegerinnenschule und Wärterinnen, welche Kranke mit offener schwerer Lungentuberkulose pflegen und mithin immer der Gefahr der Tuberkulose-Ansteckung ausgesetzt sind, sowie Nichttuberkulöse und Tuberkulöse unserer Klinik.

\section{Versuchsmethnden.}

Morgens früh vot Reinigung der Mundhöhle liess man die Versuchspersonen mit ungefähr 20-25 ccm sterilen Wassers gurgeln; das Gurgelwasser kam in ein Reagenzglas (Länge: $30 \mathrm{~cm}$, Durchmesser : 2,5 cm, Kapazität : $100 \mathrm{ccm}$ ). Wenn ein Niederschlag spontan entstand, wurden ungefähr $15 \mathrm{ccm}$ des Niederschlages mit derselben Menge einer 4\%igen Schwefelsäurelösung versetzt. Nach 20-30 Minuten langem Stehenlassen wurde das Versuchsmaterial mittels einer Zentrifuge von 3000 Umdrehungen pro Minute 10 Minuten lang zentrifugiert; der so erhaltene Niederschlag wurde sorgfältig auf 3-5 Oka-Katakura sche Phosphorsäure-Ajinomoto-Hühnerei-Nälirböden gestrichen. Die Beurteilung der Züchtungsergebnisse erfolgte in gleicher Weise wie bei dem Nasenspülwasset.

3. Versuchsergebnisse.

a) Bei Personen, die der Gefahr der Tuberkulosenansteckung ausgesetzt sind.

An 20 Pflegerinnen, 25 Schülerinnen der Schwesterschule, 70 Wärterinnen, in ins-gesamt 115 Fällen, und zwar in jedem Falle 1-3 mal haben wir die Züchtung von Tuberkelbazillen aus Gurgelwasser durchgeführt und die Resultate gewonnen, die in Tabelle 4 angegeben sind. Unter 115 Versuchspersonen konnte man nämlich bei 1 Fall $(0,8 \%)$ und zwar in $1(0,6 \%)$ von 
174 Versuchen Tuberkelbazillen aus Gurgelwasser herauszüchten. Dieser eine Fall war eine Wärterin, deren klinische Befunde, biologische Reaktionen, Röntgenbild und Untersuchungsverlauf hier kurz geschildert seien.

$$
\text { Tabelle } 4 .
$$

Kulturergebnisse der Tuberkelbazillen aus Gurgelwasser bei den Personen, die der Gefahr der Tuberkulosenansteckung ausgesetzt sind.

\begin{tabular}{l|c|c|c|c|c|c}
\hline $\begin{array}{r}\text { Züchtung aus } \\
\text { Gulgel- } \\
\text { wasser }\end{array}$ & Fälle & $\begin{array}{c}\text { Tuberkel- } \\
\text { bazillen }\end{array}$ & $\begin{array}{c}\text { Apathogene } \\
\text { säurefeste } \\
\text { Bazillen }\end{array}$ & $\begin{array}{c}\text { Zahl der } \\
\text { Versuche }\end{array}$ & $\begin{array}{c}\text { Tuberkel- } \\
\text { bazillen }\end{array}$ & $\begin{array}{c}\text { Apathogene } \\
\text { säurefeste } \\
\text { Bazillen }\end{array}$ \\
\hline Pflegerinnen & 20 & 0 & $1(5,0 \%)$ & 40 & 0 & $1(2,5 \%)$ \\
\hline $\begin{array}{c}\text { Schulerinnen der } \\
\text { Pflegerinnschule }\end{array}$ & 25 & 0 & $1(4 ; 0 \%)$ & 25 & 0 & $1(4,0 \%)$ \\
\hline Wärterinnen & 70 & $1(1,4 \%)$ & $6(8,6 \%)$ & 109 & $\mathbf{1}(0,9 \%)$ & $6(5,5 \%)$ \\
\hline Gesamt & 115 & $1(0,8 \%)$ & $8(6,8 \%)$ & 174 & $1(0,6 \%)$ & $8(4,6 \%)$
\end{tabular}

Nr: 1, M. A., 20jährige Wärterin.

Von März bis Ende April 1942 arbeitete sie als Wärterin in Zimmern nichttuberkulöser Kranker und von Mai bis Juni desselben Jahres in Zimmern Tuberkulöser.

März 1942 betrug die Tuberkulinreaktion $4 \dot{\times} 4 \mathrm{~mm}$ und die Senkungsgeschwindigkeit der toten Blutkörperchen $4 \mathrm{~mm}$ pro. Stunde. Am 13. Mai desselben Jahres wurde aber aus Gurgelwasser eine Kolonie Tuberkelbazillen auf 3 Nährböden herausgezüchtet. Später fiel indessen die Züchtung aus Gurgelwasser sowie aus Sputum negativ aus. Die Tuberkulinreaktion war im Juli und September negativ, im Röntgenbild der Lunge wurden keine Veränderungen erkannt und weder Nasen-, noch Mundhöhle, noch Kehlkopf wiesen tuberkulöse Veränderungen auf.

Das Zimmer, in dem diesc Wärterin arbeitete, war mit Tuberkulösen belegt, von denen die meisten offene schwere Lungentuberkulose hatten, bei denen Tuberkelbazillen auf Strichpräparaten aus Sputum nachgewiesen worden waren:

Apathogene säurefeste Bazillen wurden, wie aus Tabelle 4 ersichtlich ist, insgesamt bei $8(6,8 \%)$ von 115 Fällen und zwar in $8(4,6 \%)$ von 174 Versuchen aus Gurgelwasser herausgezüchtet.

b) Bei Nichttuberkulösen.

An 34 Nichttuberkulösen und zwar in jedem Falle 1-3 mal, wurden Züchtungen von Tuberkelbazillen aus Gurgelwasser durchgeführt und die Resultate erzielt, welche in Tabelle 5 gezeigt sind. Bei keinem der 54 Versuche wurden Tuberkelbazillen aus Gurgelwasser herausgezüchtet. 
Tabelle 5. Kulturergebnisse der Tuberkelbazillen aus Gurgelwasser bei Nichttuberkulösen.

\begin{tabular}{l|c|c|c|c|c|c}
\hline $\begin{array}{r}\text { Züchtung aus } \\
\text { wargel- }\end{array}$ & Fälle & $\begin{array}{c}\text { Tuberkel- } \\
\text { bazillen }\end{array}$ & $\begin{array}{c}\text { Apathogene } \\
\text { säurefeste } \\
\text { Bazillen }\end{array}$ & $\begin{array}{c}\text { Zahl der } \\
\text { Versuche }\end{array}$ & $\begin{array}{c}\text { Tuberkel- } \\
\text { bazillen }\end{array}$ & $\begin{array}{c}\text { Apathogene } \\
\text { säurefeste } \\
\text { Bazillen }\end{array}$ \\
\hline Nichttuberkulose & 34 & 0 & $3(8,8 \%)$ & 54 & 0 & $3(5,5 \%)$
\end{tabular}

Apathogene säurefeste Bazillen wurden aus Gurgelwasser bei $3(8,8 \%)$ von 34 Fällen, und zwar in $3(5,5 \%)$ von 54 Versuchen, nachgewiesen.

Aus diesen Ergebnissen geht hervor, dass in Krankenzimmern Nichttuberkulöser zwar nicht Tuberkelbazillen, wohl aber apathogene säurefeste Bazillen nachgewiesen werden.

c) Bei Tuberkulösen.

1. Beziehung zwischen den Tuberkelbazillen im Gurgelwasser und denen im Sputum.

Bei $90(64,6 \%)$ von 139 Tuberkulösen und zwar in $176(49,0 \%)$ von 361 Versuchen fiel die Züchtung von Tuberkelbazillen aus Gurgelwasser positiv aus. Die Beziehung zwischen den Züchtungsergebnissen und den Tuberkelbazillen im Sputum ist in Tabelle 6 angegeben. Oka ${ }^{9)}$ hat an unserer Klinik die Züchtung von Tuberkelbazillen aus Rachenschleim durchgeführt.

Nach ihm fiel die Züchtung von Tuberkelbazillen aus Rachenschleim bei $19(63,3 \%)$ von 30 tuberkulösen Fällen mit positivem Ausstrich aus Sputum positiv aus. Bei $1(25,0 \%)$ von 4 Fällen, denen es an Sputum fehlte, und bei denen die Tuberkulinreaktion positiv war und in deren subjektiven Symptomen, klinischen Befunden und Röntgenbild der Lunge keine Veränderungen erkannt wurden, und bei $13(43,3 \%)$ von 30 Fällen, bei denen die mikroskopische Untersuchung des Ausstrichpräparates immer negativ war, sowie bei $27(90,0 \%)$ von 30 Fällen, bei denen die mikroskopische Untersuchung des Ausstrichpräparates positiv war, wurden positive Züchtungsergebnisse von Tuberkelbazillen aus Gurgelwasser erzielt. Mikami ${ }^{103}$ hat bei $54(53,5 \%)$ von 101 Fällen, denen es an Sputum fehlte und bei denen die mikroskopische Untersuchung des Ausstrichpräparates negativ war, positive Züchtungsergebnisse von Tuberkelbazillen aus Rachenschleim gewonenn.

Nach Oyama ${ }^{11)}$ fiel die Züchtung von Tuberkelbazillen aus Magensaft bei $102(59,0 \%)$ von 173 Fällen, denen es an Sputum fehlte, bei $33(44,6 \%)$ von 74 Fällen mit negativem Ergebnis der Züchtung aus Sputum, bei 44 $(97,8 \%)$ von 45 Fällen mit positivem Ergebnis der Züchtung aus Sputum, bei $8(61,5 \%)$ von 13Fällen mit negativem Ergebnis des Ausstrichs aus Sputum, 
bei $15(100 \%)$ von 15 Fällen mit positivem Ergebnis des Ausstrichs aus Sputum, insgesamt bei $202(63,1 \%)$ von 320 Fällen positiv aus.

Tabelle 6.

Beziehung zwischen den Tuberkelbazillen im Gurgelwasser und denselben im Sputum bei Tuberkulösen.

\begin{tabular}{|c|c|c|c|c|c|c|}
\hline $\begin{array}{r}\text { Züchtung aus } \\
\begin{array}{r}\text { Gurgel- } \\
\text { wasser }\end{array} \\
\text { Sputum }\end{array}$ & $\begin{array}{l}\text { Zahl der } \\
\text { Versuche }\end{array}$ & $(-)$ & $(+)$ & (H) & (H) & $\begin{array}{l}\text { Positive } \\
\text { Ausfälle der } \\
\text { Tuberkel- } \\
\text { bazillen }\end{array}$ \\
\hline feblt & 23 & 21 & 2 & 0 & 0 & $2(8,7 \%)$ \\
\hline Kultur (-) & 91 & 79 & 12 & 0 & 0 & $12(13,2 \%)$ \\
\hline Kultur $(+) \dagger$ & 98 & 52 & 40 & 3 & 3 & $46(46,9 \%)$ \\
\hline Ausstrich $(-)^{*}$ & 23 & 19 & 4 & 0 & 0 & $4(17,2 \%)$ \\
\hline \multirow{3}{*}{ Geffky } & 54 & 13 & 23 & 6 & 12 & $41(75,9 \%)$ \\
\hline & 44 & 1 & 13 & 4 & 26 & $43(97,7 \%)$ \\
\hline & 28 & 0 & 4 & 1 & 23 & $28(100 \%)$ \\
\hline \multirow{2}{*}{$\begin{array}{l}\text { Gesamtzahl der } \\
\text { Versuche }\end{array}$} & \multirow{2}{*}{361} & \multirow{2}{*}{185} & $\underbrace{98}$ & 14 & 64 & \multirow{2}{*}{$176(49,0 \%)$} \\
\hline & & & \multicolumn{3}{|c|}{$176(49,0 \%)$} & \\
\hline Gesamt (Fälle) & 139 & 49 & \multicolumn{3}{|c|}{$90(64,6 \%)$} & $90(64,6 \%)$ \\
\hline
\end{tabular}

(+) 1-30 Kolonien der Tuberkelbazillen.

(H) 31-100 Kolonien der Tuberkelbazillen.

(H) über 100 Kolonien der Tuberkelbazillen.

+ Ausstrich immer negativ.

* Ausstrich immer negativ und Züchtung noch nicht vorgenommen.

Aus den Resultaten geht hervor, dass, wie $\mathrm{Oka}^{97}$, Mikami ${ }^{10)}$ und Oyama ${ }^{11)}$ an unserer Klinik schon mitteilten, die Tuberkelbazillen in der Mundhöhle der Tuberkulösen in einem sehr hohen Prozentsatz nachgewiesen werden können und dass die Bazillenmenge sowie der Prozentsatz der positiven Ergebnisse mit der Menge der Tuberkelbazillen im Sputum parallel laufen.

2. Beziehung zwischen den säurefesten Bazillen im Gurgelwasser und denen im Nasenspülwasser.

An 139 Tuberkulösen wurden mit der Züchtung aus Nasenspülwasser gleichzeitig parallel auch Züchtungen von Tuberkelbazillen aus Gurgelwasser durchgeführt und die Resultate erzielt, wie sie in Tabelle 7 angegeben sind. Unter 139 Tuberkulösen fiel die Züchtung von Tuberkelbazillen aus Nasenspülwasser bei 23 Fällen $(16,5 \%)$ und zwar in $31(8,5 \%)$ von 361 Versuchen positiv aus. 
Tabelle 7.

Beziefung zwischen den säurefesten Bazillen im Gurgelwasser und denselben im Nasenspülwasser bei Tuberkulösen.

\begin{tabular}{|c|c|c|c|c|}
\hline $\begin{array}{c}\text { Züchtung aus } \\
\text { Nasenspül- } \\
\text { wasser }\end{array}$ & $\begin{array}{l}\text { Zahl der } \\
\text { Versuche }\end{array}$ & Tuberkelbazillen & $\begin{array}{l}\text { Apathogene } \\
\text { säurufefeste } \\
\text { Bazillen }\end{array}$ & $\begin{array}{l}\text { Apathogene } \\
\text { säurefeste } \\
\text { Bazillen aus } \\
\text { Gurgelwasser }\end{array}$ \\
\hline$(-)$ & 185 & $5(2,7 \%)$ & $20(10,8 \%)$ & $8(4,3 \%)$ \\
\hline$(+)$ & 98 ) & \multirow{3}{*}{$\left(\begin{array}{c}26 \\
(14,6 \%)\end{array}\right.$} & \multirow{3}{*}{$\begin{array}{c}21 \\
(11,9 \%)\end{array}$} & \multirow{3}{*}{$-\int(3 ; 9 \%)$} \\
\hline$(H)$ & \multirow[t]{2}{*}{176} & & & \\
\hline (H) & & & & \\
\hline $\begin{array}{l}\text { Gesamtzahl der } \\
\text { Versuche }\end{array}$ & $361\left\{\begin{array}{l}\$ 205 \\
\$ 156\end{array}\right.$ & $(8,5 \%)\left\{\begin{array}{c}10 \\
(4,7 \%) \\
21 \\
7(13,4 \%)\end{array}\right.$ & $(11,3 \%)\left\{\begin{array}{c}\hat{\delta}(9,7 \%) \\
21 \\
21 \\
(13,4 \%)\end{array}\right.$ & $\begin{array}{c}15 \\
(4,2 \%)\end{array}\left\{\begin{array}{c}8 \\
(3,9 \%) \\
7 \\
q(4,4 \%)\end{array}\right.$ \\
\hline Gesamt (Fälle) & $139 \begin{cases}\hat{8} & 78 \\
8 & 61\end{cases}$ & $(16,5 \%)\left\{\begin{array}{c}23 \\
(11,5 \%) \\
14 \% \\
(22,9 \%)\end{array}\right.$ & $(25,8 \%)\left\{\begin{array}{c}1 \text { 웅 }(21,8 \%) \\
19 \%) \\
\text { 우 }(31,1 \%)\end{array}\right.$ & $\begin{array}{c}15 \\
(10,7 \%)\left\{\begin{array}{c}8 \\
\text { s }(10,2 \%) \\
7\end{array}\right) \\
\text { 우 }(11,4 \%)\end{array}$ \\
\hline
\end{tabular}

(t) 1-30 Kolonien der Tuberkelbazillen.

(H) 31-100 Kolonien dex Tuberkelbazillen.

(H) über 100 Kolonien der Tuberkelbazillen.

Berücksichtigt man das Geschlecht, so fiel die Züchtung von Tuberkelbazillen aus Nasenspülwasser bei $9(11,5 \%)$ von 78 männlichen Versuchpersonen, und zwar in $10(4,7 \%)$ von 205 Versuchen und bei $14(22,9 \%)$ van 61 weiblichen Versuchspersonen, und zwar in $21(13,4 \%)$ von 156 Versuchen positiv aus. Der Ptozentsatz des positiven Nachweises der Tuberkelbazillen aus Nasenspülwasser läuft mit der Menge der Tuberkelbazillen im Gurgelwasser parallel.

Apathogene säurefeste Bazillen wurden in 36 Fällen (25,8\%) von 139 Versuchspersonen und zwar in $41(11,3 \%)$ von 361 Versuchen im Nasenspülwasser nachgewiesen.

Barücksichtigt man das Geschlecht, so wurden apathogene säurefeste Bazillen im Nasenspülwasser bei 17 Fällen $(21,8 \%$ ) von 78 männlichen Versuchs-Personen, und zwar in $20(9,7 \%)$ von 205 Versuchen und bei $19(31,1 \%)$ von 61 weiblichen Versuchspersonen und zwar in $21(13,4 \%)$ von 156 Versuchen nachgewiesen.

Aus dem Gesagten kann man erkennen, dass die Züchtungsergebnisse von apathogenen säurefesten Bazillen aus Nasenspülwasser mit der Menge der Tuberkelbazillen im Gurgelwasser in keiner Beziehung stehen.

Bei 15 Fällen (10,7\%) von 139 Versuchspersonen und zwar in 15 (4,2\%) von 361 Versuchen wurden apathogene säurefeste Bazillen aus Gurgelwasser 
nachgewiesen. Die Beziehung zwischen den Resultaten und den Tuberkelbazillen im Gurgelwasser ist in Tabelle 7 dargestellt.

Berücksichtigt man das Geschlecht, so zeigten 8 Fälle $(10,2 \%)$ von 78 männlichen Versuchspersonen und zwar $8(3,9 \%)$ von 205 Versuchen, und $7(11,4 \%)$ von 61 weiblichen Versuchspersonnen, und zwar $7(4,4 \%)$ von 156 Versuchen positives Ergebnis.

Aus den Ergebnissen geht hervor, dass apathogene säurefeste Bazillen im Gurgelwasser in sehr geringer Anzahl vorhanden sind und mit den Tuberkelbazillen im Gurgelwasser in keiner Beziehung stehen.

\section{Die Züchtung von Tuberkelbazillen aus Staub in der Luft von Krankenzimmern Tuberkulöser.}

\section{Versuchsmaterial und-methode.}

Bei einigen Massenuntersuchungen des Sommers fügte $A \circ \mathrm{ki}^{12)} \mathrm{dem}$ Sputum eine 0,3-0,5\% ige Schwefelsäurelösung hinzu, um dem Verfaulen des Materials durch Saprophyten bei einer langen Beförderung vorzubeugen, und konnte ein ganz befriedigendes Ergebnis erzielen. In unseren Versuchen kamen ungefähr $300 \mathrm{ccm}$ einer $0,5 \%$ igen Schwefelsäurelösung in eine Entwicklerschale (Länge : $37,5 \mathrm{~cm}$, Breite $: 33 \mathrm{~cm}$, Tiefe $: 5 \mathrm{~cm}$ ) und wurden in eine Ecke des Zimmers der Tuberkulösen gestellt. Nach 1, 2 und 5 Tage langem Stehenlassen kamen je ungefähr $20 \mathrm{ccm}$ des Versuchsmaterials in ein steriles Spitzglas und nach Zugabe von ungefähr $15 \mathrm{ccm}$ einer $4 \%$ igen Schwefelsäurelösung wurde es 30 Minuten lang bei Zimmertemperatur stehen gelassen. Dann wurde es mittels einer Zentrifuge mit 300 Umdrehungen pro Minute 10 Minuten lang zentrifugiert und die überstehende Flüssigkeit weggegossen. Der Niederschlag kam auf 3-5 Oka-Katakurasche Nährböden und wurde $z$ wei Monate lang im Brutschrank bei $37^{\circ} \mathrm{C}$ kultiviert. Die ganze Flüssigkeit wurde auf gleiche Weise untersucht.

Tabelle 8.

Kulturergebnisse von Tuberkelbazillen aus Staub in der

Luft des Krankenzimmers der Tuberkulösen.

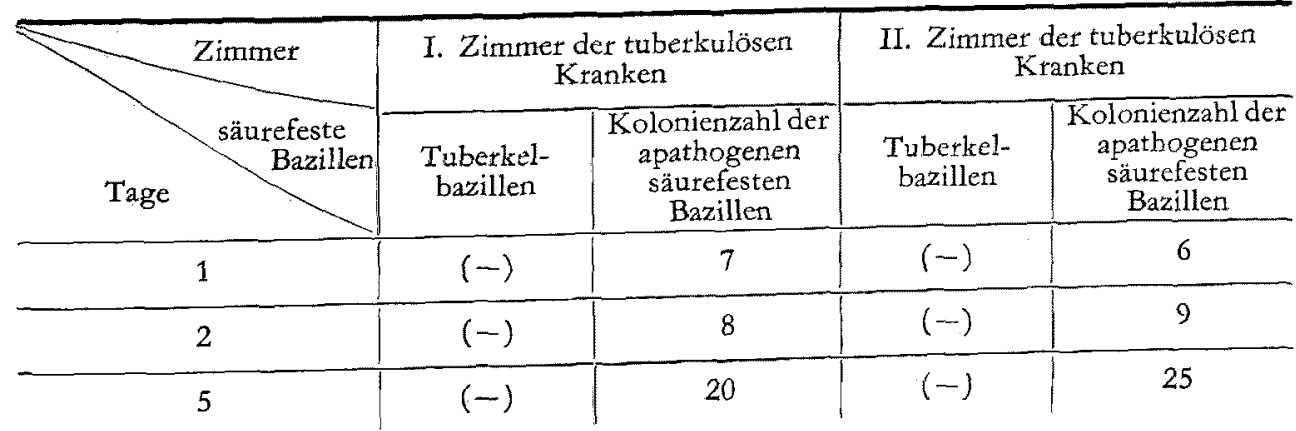




\section{Versuchsergebnisse.}

Wie aus Tabelle 8 ersichtlich ist, wurden ziemlich viele apathogene säurefeste Bazillen nachgewiesen. Aus dem Staub des 1. Zimmers, das mit 10 Tuberkulösen belegt war, wurden nach 1,2 und 5 Tage langem Stehenlassen 7, 8 bzw. 20 Kolonien apathogener säurefester Bazillen auf je 40-50 Nährboden und aus dem Staub des 2. Zimmers, das mit 5 Patienten belegt war, 6,9 bzw. 25 Kolonien herausgezüchtet.

Daraus kann man schliessen, dass verhältnismässig viele apathogene säurefeste Bazillen in der Luft vorhanden sind und dass die in der Nasenhöhle nachgewiesenen apathogenen säurefesten Bazillen aus der Luftherrührten.

Aus dem Staub der beiden Krankenzimmer wurden aber keine Tuberkelbazillen herausgezüchtet.

\section{Versuche an Meerschweinchen zur Spontan-Infektion mit Tuberkulose in Zimmern, in denen Gefahr der Tuberkulose-Infektion besteht.}

\section{Versuchsmaterial.}

Als Versuchstiere dienten 16 Meerschweinchen, 8 Männchen und 8 Weibchen von ungefähr $400 \mathrm{~g}$ Körpergewicht, die auf Tuberkulin negativ reagier$t_{\text {en: }}$

2. Versuchsmetbode.

Um die spontane Tuberkulose-Ansteckung in Zimmern zu untersuchen, wurden folgende Versuche angestellt. Mit Rücksicht auf die Lüftung, freie Bewegung der Tiere usw. wurde, um Ansteckung unter einander zu vermeiden, je 1 Tier in einem Behälter aus Drahtnetz $(22 \times 22 \times 22 \mathrm{~cm})$ gehalten. Dabei wurde der Wartmhaltung der Fütterung, usw. grosse Sorgfalt geschenkt.

Bei 8 von 16 Meerschweinchen verwandelte man die negative Tuberkulinreaktion durch intracutane, subcutane und intravenöse Injektion von 0,1 mg BCG in eine positive: Die Versuchstiere wurden in 5 Gruppen geteilt. Die 1. Gruppe umfasste 4 Paare, und je 1 Paar wurde zwischen die Betten in ein Zimmer gestellt, das mit 10 an offener Tuberkulose Erkrankten belegt war. Die 2. Gruppe (1 Paar) wurde zu Häupten der Patienten in ein Zimmer gestellt, in dem sich $5 \mathrm{Kranke}$ mit offener Tuberkulose befanden. Die 3. Gruppe (2 Paare) in das Behandlungszimmer, in dem viele Tuberkulöse einund ausgingen. Die 4. Gruppe (1 Paar) in das Zimmer der Tuberkelbazillenzüchtung. Die 5. Gruppe (1 Paar) in das Untersuchungszimmer der Substanzen, in denen Tuberkelbazillen enthalten sind.

Alle 10 Tage wurde nach der Fütterung im Zimmer die Intracutanreak- 
tion von $0,1 \mathrm{ccm}$ Alttuberkulin in der Verdünnung von $1: 5$ durchgeführt. Nach dem Tode der Versuchstiere wurden alle ihre Organe makroskopisch untersucht, histologische Präparate hergestellt und Züchtungen von Tuberkelbazillen aus allen Organen und Lymphdrüsen durchgeführt, um festzustellen, ob eine Tuberkulose-Infektion erfolgt sei oder nicht.

3. Versucbsergebnisse.

Die mit BCG behandelten Versuchstiere der 1.-5. Gruppe lebten 47-101 Tage, und die nicht mit BCG behandelten 65-107 Tage. Darum konnte man keinen bedeutenden Unterschied zwischen den mit BCG behandelten und den nicht so behandelten Versuchstieren feststellen. Bei der Sektion sämtlicher Tiere konnten weder maktoskopisch noch histologisch tuberkulöse Veränderungen festgestellt werden. Die Züchtung von Tuberkelbazillen fiel aus allen Organen und Lymphdrüsen negativ aus. Die Resultate sind in Tabelle 9 angegeben. Wenn auch die spontane Tuberkulose-Infektion nicht nachgewiesen werden konnte, weil die Meerschweinchen nur kurz Zeit lebten, kann man doch Spontan-Infektion durch Tuberkulose im Zimmer nịcht durchaus verneinen.

Aus den Züchtungsergebnissen von Tuberkelbazillen aus dem Staub der Zimmer von tuberkulösen Kranken sowie aus den an Meerschweinchen gewonnenen Resultaten der Versuche zur Spontan-Infektion mit Tuberkulose in Zimmern, in denen Gefahr der Tuberkulose-Infektion besteht, kann man schliessen, dass Tuberkelbazillen selbst in Zimmern, die Kranke mit offener schwerer Lungentuberkulose beherbergt, nur wenig vorhanden sind und sich nicht leicht nachweisen lassen, wenn man die Fenster des Zimmers bei Tag und Nacht offen lässt.

\section{Zusammenfassung und Betrachtung.}

Wenn die Ansteckung mit Tukerkulose, wie man im allgemeinen glaub̧t, durch die áerogenen Wege erfolgt, so müssten in Nase oder Rachen und Kehlkopfschleimhaut der Personen, die unter Gefahr der, Tuberkulose-Infektion leben, oft Tuberkelbazillen nachgewiesen werden können, welche von dex Inspiration herrühren. Um diesen Sachverhalt zu prüfen, haben wir Tuberkelbazillen aus Nasenspül- und Gurgelwasser von Ärzten, Pflegerinnen, Wärterinnen usw., welche mit an offener schwerer Lungentuberkulose Erkrankten in Berührung kommen, isoliert und kultiviert.

Bei 293 Versuchen, die mit 147 der Gefahr der Tuberkulose-Infektion ausgesetzten Versuchspersonen angestellt wurden, wurden 'Tuberkelbazillen aus Nasenspülwasser bei 2 Personen $(1,3 \%$ ) herausgezüchtet. Bei 174 Versuchen, die mit 115 Versuchspersonen angestellt wurden d.h. mit Pflegerinnen, Schülerinnen der Pflegerinnenschule und Wärterinnen, welche unter 


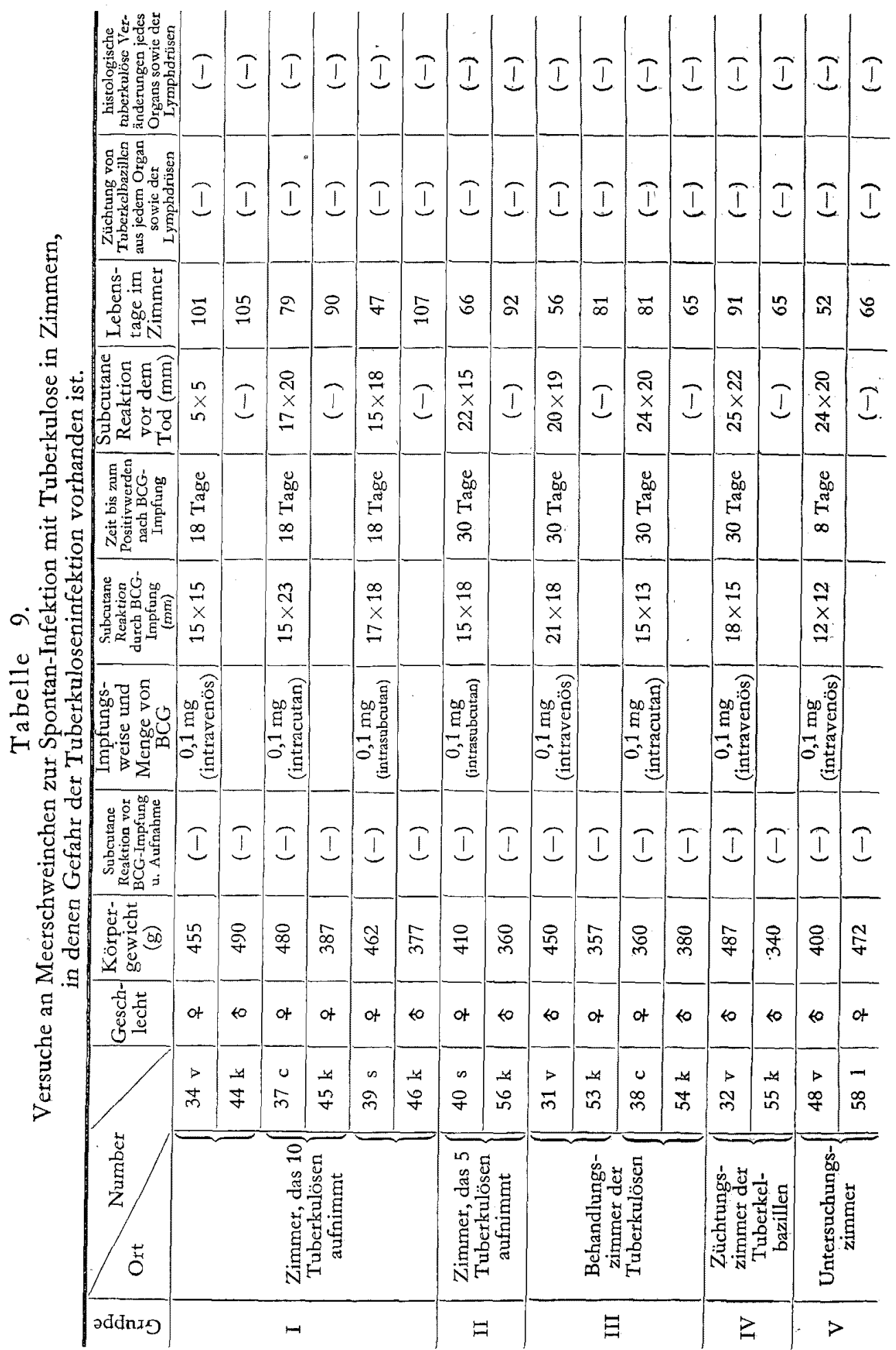


der Gefahr der Tuberkulose-Infektion arbeiten, wurden Tuberkelbazillen aus Gurgelwasser bei 1 Fall $(0,8 \%)$ nachgewiesen.

Bei den 2 Fällen mit positivem Züchtungsergebnis aus Nasenspülwasser war die Tuberkulinreaktion positiv, aber mit Rücksicht auf Anamnese, subjektive Symptome, objektive Befunde der Lunge, biologische Untersuchungen, Röntgenbild der Lungen, Prognose und Befunde in der Nasen- und Mundhöhle, im Rachen und Kehlkopf konnte man eine tuberkulöse Errkrankung durchaus verneinen. Darum kann man annehmen, dass die nachgewiesenen Tuberkelbazillen solche waren, die durch Inspiration eingeatmelt, an der Nasenschleimhaut hafteten.

Bei dem 1 Fall mit positivem Züchtungsergebnis aus Gurgelwasser war die Tuberkulinreaktion negativ, und die Züchtung aus Sputum fiel immer negativ aus. Darum können die nachgewiesenen Tuberkelbazillen ebenfalls als exogene Bazillen betrachtet werden. Dazu kommt noch, dass die Tuberkelbazillen bei diesen Fällen nicht fotwährend nachgewiesen wurden und auch nur eine Kolonie entstand.

J. Strau s $^{4}$ untersuchte die Tuberkelbazillen im Nasenschleim derjenigen Personen, die unter grosser Gefahr der Tuberkulose-Ansteckung lebten, und bekam in Tierversuchen positive Resultate, was aber Le Noir und J. Camus ${ }^{5)}$ nicht gelang. Aus den Ergebnissen unserer Versuche, in denen die Tuberkelbazillen, wenn auch selten, in der Nasen- und Mundhöhle nachgewiesen wurden, lässt sich aber schliessen, dass die aerogene Infektion für die spontane Ansteckung durch Tuberkulose eine gewisse Rolle spielt. Der Nachweis der Tuberkelbazillen ist dabei selbstverständlich davon abhängig, wie unrein die Luft des Zimmers ist, ob die Fenster des Krankenzimmers immer offen sind, wie es mit dem Befinden der Patienten steht und nach welcher Methode die Tuberkelbazillen nachgewiesen werden.

Als Kontrollversuch haben wir Nasenspülwasser von 78 Nichttuberkulösen und Gurgelwasser von 54 Nichttuberkulösen auf Tuberkelbazillen untersucht. Aber in keinem Falle wurden solche nachgewiesen. Aus dieser Tatsache kann man schliessen, dass die Möglichkeit der TuberkuloseInfektion sehr abhängig von dem Milieu ist.

Wir haben ferner Züchtung det Tuberkelbazillen aus dem Staub in der Luft durchgeführt. E. Bogen und W. Dunn ${ }^{13}$ ) teilten mit, dass ihnen der Nachweis von Tuberkelbazillen aus Staub in Zimmern von Tuberkulösen durch Kultur oder Tierversuch gelang, dass aber die Spontan-Infektion mit Tuberkulose bei Meerschweinchen schwer fiel. Maeda und Nakai ${ }^{\mathbf{1 4}}$ ) gelangten zu derselben Ansicht. Aber H. Sewall und B. Lurie ${ }^{15)}$, Sigenobu. ${ }^{16)}$, Minami ${ }^{17)}$ u.a. gelang nach ihren Angaben spontane Tuberkuloseinfektion. 
Wir konnten weder durch Kultur noch durch Tierversuch Tuberkelbazillen aus dem Staub der Zimmer von Tuberkulösen nachweisen. Daraus konnten wir schliessen, dass in der Luft nur wenige Tuberkelbazillen schweben und ihr Nachweis daher schwer ist.

Auch die Tatsache, dass im Schleim der Nasen- und Mundhöhle, die als Reinigungsapparat von Staub und Bakterien betrachtet werden können, und im Nasenspül- und Gurgelwasser nur selten Tuberkelbazillen gefunden werden, steht unseres Erachtens in gewissem Zusammenhang damit, dass wie oben erwähnt, die Dichtigkeit der Tuberkelbazillen in der Luft sehr gering ist.

Aus der Tatsache, dass exogene Tuberkelbazillen im Nasenspül- oder Gurgelwasser nachgewiesen werden, wird darauf geschlossen, dass es vonnöten ist, bei der Züchtung der Tuberkelbazillen aus Sputum, Magensaft oder Rachenschleim, insbesondere bei Massenuntersuchungen die jenigen Fälle besonders genau zu untersuchen, bei denen, wenn auch ohne subịektiv Beschwerde und pathologische Befunde im Röntgenbild, doch die Züchtung der Tuberkelbazillen positiv ausfällt. Bei solchen Fällen sind biologische Reaktionen und fortdauernde Kultur der Tuberkelbazillen durchzuführen und genau zu prüfen.

Wir haben ferner die Züchtung von Tuberkelbazillen aus Nasenspül- und Gurgelwasser ausgeführt. Bei 473 Versuchen, die mit 142 Tuberkulösen angestellt wurden, fiel die Züchtung aus Nasenspülwasser bei 28 Fällen positiv aus, allein bei keinem von ihnen wurden tuberkulöse Veränderungen in der Nasenhöhle konstatiert. Daraus ist zu erkennen, dass die Nasentuberkulose sehr selten ist. Sekin ${ }^{18)}$ teilt ebenfalls mit, dass die Nasentuberkulose sehr selten ist, weil er sie nur bei ungefähr $0,5 \%$ von 1048 Lungentuberkulösen wahrgenommen hat.

Bei 361 Versuchen, die mit 139 Tuberkulösen angestellt wurden, fiel die Züchtung von Tuberkelbazillen aus Gurgelwasser in 90 Fällen $(64,6 \%)$ positiv aus. Daraus wird geschlossen, dass die Tuberkelbazillen oft in der Nasenhöhle oder dem Rachenschleim von Tuberkulösen haften.

Da positive Resultate der Bazillenmenge und der Auswurfsmenge parallel laufen, ist es denkbar, dass die nachgewiesenen Tuberkelbazillen von den Patienten selbst herrührten.

Unter 123 Versuchen an Tuberkulösen, bei denen die Züchtung von Tuberkelbazillen aus Sputum negativ ausgefallen war, wurden indessen bei 4 Fällen (3,3\%) Tuberkelbazillen im Nasenspülwasser nachgewiesen und unter 186 Versuchen an Versuchspersonen, bei denen die Versuche aus Gurgelwasser negativ waren, wurden sie in 5 Fällen $(2,7 \%)$ im Nasenspülwasser nachgewiesen. In diesen positiven Fällen ist schwer fetszustellen, ob die Tuberkelbazillen in der Nasenhöle von den Patienten selbst herrühtten oder 
Kulturelle Nachweis von Tuberkelbazillen im Nasenspülwasser

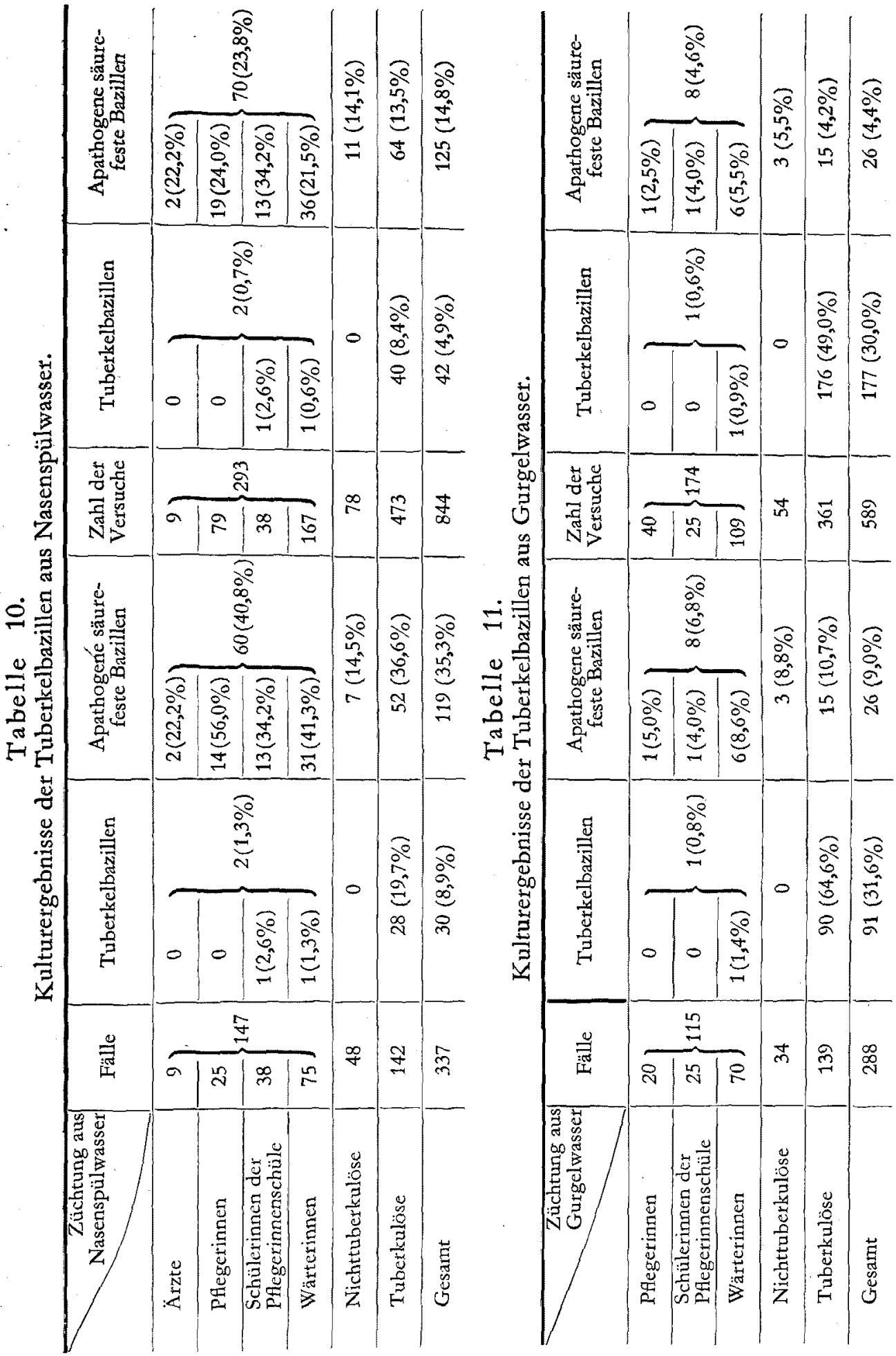


durch Inspiration eingeatmete, exogene Tuberkelbazillen waren.

Im Nasenspül- und Gurgelwasser wurden ferner bei der Züchtung der Tuberkelbazillen auch apathogene säurefeste Bazillen in einem sehr hohen Prozentsatz gefunden. (Tabelle 10, 11)

Bei $119(35,3 \%)$ von 337 Versuchspersonen und zwar in $125(14,8 \%)$ von 844 Versuchen, bei denen die Züchtung der Tuberkelbazillen aus Nasenspülwasser ausgeführt worden war, und bei $26(9,0 \%)$ von 288 Versuchspersonen, und zwar in $26(4.4 \%)$ von 589 Versuchen, bei denen dieselbe aus Gurgelwasser ausgeführt worden war, wurden apathogene säurefeste Bazillen nachgewiesen. Die apathogenen säurefesten. Bazillen, die im Schleim der Mundhöhle gehaftet hatten, können ausser vom Luftweg auch davon herrühren, dass sie mit der Nahrung zusammen hereinkamen. Die in der Nasenhöhle aber werden meist vom Luftweg hergekommen sein.

Andererseits wurden sehr viele apathogene säurefeste Bazillen im Staub der Luft gefunden. Daraus konnte man erkennen, dass die apathogenen säurefesten Bazillen im Staub der Luft mit denen im Nasenspül- oder Gurgelwasser in enger Beziehung stehen.

Die oben erwähnten Resultate liefern einen Bewies dafür, dass die aerogene Infektion bei der spontanen Infektion der Tubexkulose eine bedeutende Rolle spielt.

\section{Schluss.}

1) Bei 2 von 293 Versuchen an 147 Versuchspersonen, welche Kranke mit offener schwerer Lungentuberkulose ärztlich untersuchen und behandeln oder pflegen und mithin der Gefahr der Tuberkulose-Ansteckung ausgesetzt sind, fiel die Züchtung von Tuberkelbazillen aus Nasenspülwasser positiv aus. Und bei 1 von 174 Versuchen in 115 Fällen, welche ebenfalls derselben Gefahr ausgesetzt sind, fiel die aus Gurgelwasser positiv aus. Insgesamt wurden nämlich bei 3 Fällen positive Züchtungsergebnisse von Tuberkelbazillen erzielt.

2) Als Kontrollversuch wurde die Züchtung der Tuberkelbazillen aus Nasenspülwasser in 78 Versuchen an 48 nicht-tuberkulösen Patienten und die aus Gurgelwasser in 54 Versuchen an 34 nicht-tuberkulösen Patienten unserer Klinik durchgetührt. Bei keinem Falle wurde ein positives Ergebnis erzielt.

3) Bei 28 Fällen von 473 Versuchen an 142 Tuberkulöscn fiel die Züchtung von Tuberkelbazillen aus Nasenspülwasser positiv aus, und bei 90 Fällen von 361 Versuchen an 139 Tuberkulösen fiel die aus Gurgelwasser positiv aus. Die nachgewiesenen Tuberkelbazillen rührten vom Sputum der Patienten selbst her. 
4) Bei 30 von insgesamt 337 Versuchspersonen wurden Tuberkelbazillen im Nasenspülwasser nachgewiesen, aber bei keinem Falle wurden tuberkulöse Veränderungen in der Nasenhöhle wahrgenommen. Daraus kann man erkennen, dass Nasentuberkulose sehr selten ist.

5) Aus dem Staub in Zimmern tuberkulöser Kranket konnte man keine Tuberkelbazillen herauszüchten, aber doch viele apathogene säurefeste Bazillen nachweisen.

6) Aus Gurgelwasser, insbesondere aus Nasenspülwasser von Versuchspersonen, welche der Gefahr der Tuberkulose-Ansteckung ausgesetzt sind, und aus denselben Tuberkuloser sowie Nichttuberkulöser konnte man viele apathogene säurefeste Bazillen nachweisen.

7) Aus den oben erwähnten Resultaten ist zu erkennen, dass die acrogene Infektion bei der spontanen Infektion der Tuberkulose eine bedeutende Rolle spielt.

Diese Forschung wurde durch Überlassung von Geldmitteln des Unterrichtsministeriums für wissenschaftliche Forschung ausgefühtt, wofür herzlich gedankt sei.-Prof. Dr. T. Kumagai und Prof. Dr. T. Ebina.

\section{Literatur.}

(1) Cornet, Z. Hyg., 1889, 5, 191.

(2) Flügge, Z. Hyg., 1899, 30, 107.

(3) Deycke, Praktisches Lehrbuch d. Tuberkulose, Berlin, 1920, 40.

(4) Straus, Arch. Méd. expér., 1894, 6, 460.

(5) Noir et Camus, C. R. Soc. Biol., 1908, 65, 622.

(6) Meyer, La tuberculose pulmonaire occulte à expectoration bacillifere, Legrand, Paris, 1935. (Zit. nach A. Calmette. L'infection Bacillaire et Tuberculose, Paris, 1936, 274.)

(7) Kato, Tohoku Igaku Zassi, 1943, 33, 16.

(8) Oka u. Katakura, Nippon Rinsyo Kekkaku, 1940, 1, 829.

(9) Oka, Kekkaku, 1939, 17, 466.

(10) Mikami, Kekkaku, 1940, 18, 1166.

(11) Oyama, Tohoku J. exp. Med., 1941, 39, 505.

(12) A ok i, Kekkaku, 1942, 20, 10.

(13) Bogen a. Dunn, Amer. Rev. Tbc., 1941, 43, 435.

(14) Maeda u. Nakaie, Kekkaku, 1928, 6, 20.

(15) Sewall a. Lurie, Amer. Rev. Tbc., 1924, 9, 525.

(16) Sigenobu, Kekkaku, 1930, 8, 505.

(17) Minami, Kekkaku, 1925, 4, 532.

(18) Sekine, Dainihon Zibi. Kh., 1930, 35, 1437. 